\title{
artículos
}

\section{Aportación al entorno del Museo Carmen Thyssen Málaga: Un análisis urbanístico del espacio urbano a finales del siglo XV}

\section{Ma Victoria García Ruiz}

Investigadora vinculada a la UMA

\section{RESUMEN}

En este artículo analizamos el espacio urbano que ocupa el Museo Carmen Thyssen Málaga, partiendo de la información que se recoge en los Libros del Repartimiento de Málaga.

PALABRAS CLAVE: Urbanismo de Málaga/ calles Compañía y Mártires/ Museo Carmen Thyssen Málaga.

A contribution to knowledge of Carmen Thyssen's Museum urban space in Málaga

ABSTRACT

This article looks at the urban space occupied by the Museo Carmen Thyssen Málaga, based on the information contained in Libros del Repartimiento de Málaga.

KEY WORDS: Urbanism in Málaga/ Compañía Street and Mártires Street/ Museo Carmen Thyssen Málaga.

Sin duda, el entorno del Museo Carmen Thyssen Málaga se inserta en una de las zonas más significativas del entramado medieval, y el hecho de que su fundación haya despertado el interés de la ciudadanía por conocer la historia y evolución del espacio urbano que ocupa, justifica nuestra contribución.

Como ya se sabe, del total de edificios que integran el conjunto museístico, ocupa un lugar relevante el Palacio de Villalón, que data de mediados del siglo XVI y conserva un conjunto espléndido de armaduras de lazo y adornos de yeserías'.

* GARCÍA RUIZ, Ma Victoria: "Aportación al entorno del Museo Carmen Thyssen Málaga: Un análisis urbanístico del espacio urbano a finales del siglo XV", en Boletín de Arte $\mathrm{n}^{\circ}$ 32-33, Departamento de Historia del Arte, Universidad de Málaga, 2011-2012, págs. 311-321. Fecha de recepción: Julio de 2011.

1 Así consta en CAMACHO, R., (Dir.), Guía Histórico-Artística de Málaga, Málaga, 2006, 157, 163 y 344 donde también se apunta la posibilidad de que la Casa-Palacio de los Villalón sea la misma que en el archivo Temboury se denomina de "Mosquera".

2 Documento elaborado por los responsables del proyecto de rehabilitación, ROLDÁN MATEO, R., y GONZÁLEZ GARCÍA, J., "El Museo Carmen Thyssen-Bornemisza de Málaga", Viva la calle. Las actuaciones de recuperación integral del centro histórico de Málaga desde 1994 a 2010, Málaga, 2011, disponible en http:// programaseuropeos-malaga.com/subidas/archivos/arc_2191.pdf.

3 Fundamentalmente, noticias aparecidas en la prensa local. Véase, por ejemplo, GUILLÉN, A. "Villalón incorporará al Museo los restos industriales romanos", La Opinión de Málaga, 25 de marzo de 2007, disponible en http://www.laopiniondemalaga.es/luces-malaga/2838/villalon-incorporara-museo-restos-industrialesromanosb/107557.html; GUTIERREZ, F., "Lo que esconde el Thyssen", Diario Sur, 28 de diciembre de 2009, disponible en http://www.diariosur.es/20091228/cultura/esconde-thyssen-20091228.html. 
Sobre el Palacio de Villalón hay alguna información desde la perspectiva aquitectónica ${ }^{2}$, arqueológica ${ }^{3}$ y de la propia familia Villalón ${ }^{4}$, pero nada se dice sobre la evolución que experimentó el Palacio Villalón y su entorno desde la conquista de la ciudad en 1487 por los Reyes Católicos, cuando se procede a distribuir los bienes urbanos entre los repobladores cristianos. Por consiguiente, nuestro interés se va a centrar en desentrañar el número de familias que recibieron casas en el espacio urbano que ocupa el Museo, partiendo de esa maravillosa fuente que son Los Libros del Repartimiento de Málaga $^{5}$, sobre los que se ha dicho tan acertadamente que son "un auténtico registro notarial de la propiedad" y vienen a ser, en definitiva, "una auténtica partida de nacimiento de la Málaga moderna"6.

Interesa, por tanto, no solo saber quién fue el beneficiario del Palacio de Villalón, sino también quiénes fueron los beneficiarios de los solares que actualmente conforma el ámbito del Museo Carmen Thyssen Málaga porque hay que tener presente que el magnífico complejo museístico se ha conformado mediante la integración de varios solares: en la calle de San Telmo, el número 11; en la calle de los Mártires, los números 2, 4, 5, 6 y 8; en la calle Compañía, los números 6, 8, 10 y 12; y en la Plaza de San Ignacio, el número 4 (véase [1]).

Tenemos que trasladarnos, necesariamente, a 1493, año en el que se aborda la reformación del Repartimiento por el bachiller Juan Alonso Serrano, cuando habían transcurrido más de cinco años desde la conquista de la ciudad y ya se había distribuido el suelo entre los primeros repobladores. Durante esos años la trama urbana heredada de la Málaga musulmana había sufrido pocas transformaciones en el entorno de la actual calle de los Mártires; incluso, puede afirmarse que en la actualidad la zona sigue conservando en lo esencial la traza medieval.

El sector que estudiamos se articulaba mediante dos vías principales: la primera, denominada por los castellanos calle de las Guardas ${ }^{7}$ (actual Compañía), era una de las vías más importantes de la ciudad en época musulmana, pues prolongándose por las calles Mercaderes (actual calle Santa María) y Alcázar (actual calle Cister) se conformaba un eje que dividía la medina en dos partes, atravesándola de oeste a este. Y mayor relevancia cobró la calle Guardas cuando en 1494 se abrió la Puerta Nueva, que

4 El escritor Antonio Lara sitúa el origen del palacio en una poderosa familia, los Villalón, naturales de Ronda y originarios de Setenil (Cádiz), recogiendo la historia del inmueble desde el siglo XVIII, cuando cobra verdadera importancia "gracias al casamiento en 1707 de Catalina Victoria de Villalón y Mendoza con Gaspar de Bracamonte y Zapata, IV marqués de Fuente EI Sol: LARA VILLODRES, A., "La antigua casa de Villalón, hoy convertida en el Museo de Arte Carmen Thyssen”, El avisador malagueño, ${ }^{\circ}{ }^{54}$, abril 2011, disponible en http://elavisador.es/.

5 Transcritos por BEJARANO ROBLES, F., Los Repartimientos de Málaga, Málaga, vols. I, II, III y V, Málaga 1985-2000. En adelante citaremos LR.

6 Así los ha definido José Enrique López de Coca Castañer en el Prólogo a Los Repartimientos de Málaga: LR, I, VIII. Efectivamente, los Libros del Repartimiento permiten conocer quiénes fueron los beneficiarios de todos los solares de la ciudad, como así se ha analizado en GARCÍA RUIZ, Ma V., Estructura urbana de Málaga y trasformaciones tras su conquista, Tesis Doctoral inédita, Universidad de Málaga, 2009, Documento $\mathrm{n}^{\circ} 7$.

7 "La calle que se aparta de la plaça de las Quatro Calles en derecho de cal de Mercaderes hasta el adarve, cal de las Guardas”: LR, vol. I, 85. Sobre la identificación de las calles, GARCÍA RUIZ, Mª V., Málaga en 1487, Málaga, 2009, 64 
1. Reconstrucción parcelaria en 1493.

Fuente: elaboración propia.

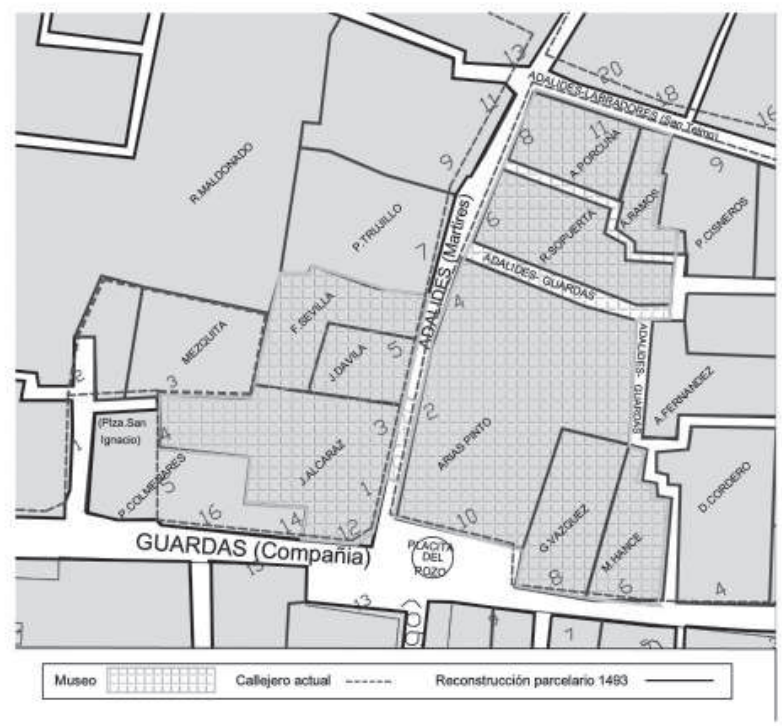

permitió poner en comunicación la Plaza Mayor (actual Plaza de la Constitución) con la zona del Guadalmedina, lo que facilitaba el acceso directo de transeúntes y mercancías hasta el mismo centro de la ciudad. La otra vía principal que articulaba el sector era la calle de Adalides $^{8}$ (actual calle de los Mártires), que de norte a sur comunicaba la calle Labradores (actuales Andrés Pérez y Santa Lucía) con la calle Guardas.

Así mismo, existía otra vía secundaria que, sin embargo jugaba un papel importante en la articulación del sector. Nos referimos a una calleja que se adentraba por la calle de Adalides y salía por la de Labradores (o viceversa), que por ello hemos denominado calleja de Adalides-Labradores, que no es otra que la actual calle de San Telmo, donde se encuentra la parte trasera del Museo. Además de estas calles principales y secundarias, existían otras callejas o barreras, ya desaparecidas, que se ramificaban por el interior de las manzanas y cuyo trazado hemos dibujado siguiendo las anotaciones contenidas en los Libros del Repartimiento. Una de ellas, que denominamos Adalides-Guardas, enmarcaba la casa que hoy conocemos como Palacio de Villalón?

Tras este breve resumen del sistema viario de la zona, vamos a detenernos en el vecindario de aquel espacio siguiendo el recorrido que realizaron los repartidores a principios de 1493 durante la visitación de las casas $^{10}$ :

8 "Otra calle que se aparta desta de Labradores a la mano izquierda, que va a dar a un pozo, do labra un herrador, calle d'Adalides": LR, vol. I, 85.

9 Las callejas Adalides-Labradores y Adalides-Guardas se integran en una de las zonas que en su momento tomamos como ejemplo para explicar la metodología seguida en la reconstrucción del parcelario de Málaga. Cf. GARCÍA RUIZ, Mª V., Málaga en 1487..., 67-68.

10 LR, vol. II, 147 y $149-150$. 
Barrera Adalides-Labradores (San Telmo)

- "La casa de Pedro de Cisneros; quédale según su asyento syn la cámara e palacio que se sacó para Alonso Ramos, yerno de Alonso de Porcuna, e él lo dio e trocó con su suegro [...] alinda con Andrés de Covarruvias y con Pedro de Cisneros y con Alonso de Porcuna" [San Telmo, 9 y 11].

Calle de los Adalides (Mártires) y barrera Adalides-Guardas (desaparecida)

- "Bolviendo por esta calle [San Telmo] a la mano ysquyerda, se vio la casa de Alonso de Porcuna" [Mártires, 8].

- Junto a la anterior "se vio la casa de Andrés de Covarruvias [...] Diose a Rodrigo de Sopuerta según está adelante" [Mártires, 6].

- "En el cabo de una barrera que junta con esta casa [de Rodrigo de Sopuerta] está la casa de maestre Pero, artillero de sus altezas. Vídose; quédale según su asyento y donación".

- Junto a la anterior, "en el ryncón de la dicha barrera, está la casa de Alonso Fernández, odrero. Vídose; quédale según su asiento”.

- junto a la anterior, "en el dicho rincón, se vio la casa de Arias Pynto, que tiene su puerta principal a la calle de las Guardas" [Mártires, 4 y 2].

Calle de las Guardas (Compañía)

- La casa de Arias Pinto, "ase de ver el libro e su asiento; vídose; quédale proveydo según el asiento de la dicha primera donación, que alinda con Alonso Fernández, odrero , e con casa de García Vázquez" [Compañía, 10 -Palacio de Villalón-].

- "Syguiendo por la calle de las Guardas hazia la plaça mayor, se vio la casa de García Vázquez, questá junto con ella; está asentado por escudero; es recuero e trabajador; está para ver y averse de proveer lo que fuere justo dello según su calidad, e lo otro proveello [Compañía, 8].

- Junto a la anterior "está la casa de maestre Hance; no se falló asiento porque es de merced; dize que tiene donación; hase de ver; vídose e quédale proveyda [Compañía, 6].

Calle de los Adalides (Mártires)

- "Tornando por la calle de Adalides se vio la casa de Juan de Alcaraz, que es de frente a la plaçuela; quédale según su asiento con el cargo de la obra e labor que tiene fecho de nuevo. Alinda con Pero de Colmenares e con Juan Dávila, organysta" [Compañía, 12-Mártires 1-3].

- “Junto con ésta está la casa de Juan Dávila, organista; parece por el asyento 
que tiene dos cuerpos; quédanle dos cuerpos según su donación. Junto con ésta se vio la casa de Francisco de Sevylla; quédale según su asyento" [Mártires, 5].

Tras este breve recorrido por las parcelas que integran el conjunto museístico, considero oportuno detenernos en cada uno de los solares.

\section{San Telmo, 11 y Mártires, 8.}

El número 8 de la calle de los Mártires y 11 de la calle de San Telmo lo ocupaban el escudero Alonso de Porcuna y su yerno el albañil Alonso Ramos, si bien ambas familias no recibieron sus casas al mismo tiempo. El primero en tomar posesión de su vivienda fue Alonso de Porcuna, que se avecindó el 15 de julio de 1489 declarando que traía caballo y armas, así como cincuenta mil maravedís en muebles y dineros, y el 25 de ese mismo mes recibía una casa en la calle de Adalides, con un pedazo de corral a sus espaldas ${ }^{11}$. Unos años después, en el transcurso de la reformación llevada a cabo por el bachiller Juan Alonso Serrano, Alonso Ramos solicitaba casa y heredades de vecindad declarando que llevaba un año en la ciudad residiendo con su mujer, y a principios de 1493 tuvo la suerte de recibir una casa pequeña junto a la de su suegro, conformada mediante una cámara y un palacio ${ }^{12}$ que quitaron de las casas de Pedro de Cisneros, cestero, que los ocupaba indebidamente ${ }^{13}$.

\section{MÁrtires, 6.}

El número 6 de la calle de los Mártires pasó por varios beneficiarios ${ }^{14}$ antes

11 La casa se había donado antes, en 13 de enero de 1489, a Cristóbal de Morillo, maestre de la caravela Helechina, que al poco tiempo se fue de la ciudad: "Este dicho día se dio a Christóval de Morillo, maestre de la dicha caravela [Helechina], una casa que es en la cal de Adalides, enfrente del comendador gallego, con un pedaço de corral que está a las espaldas de la dicha casa, çerrándose según está señalado entre él e Sebastián de Bega \Quitose a éste e diose a Alonso de Porcuna en XXV de jullio de LXXXIX": LR, vol. I, 137 y 410 .

12 LR, vol. II, 147; vol. V, 229 y 248. En realidad, Alonso Ramos trocó con su suegro las habitaciones que se quitaron de las casas de Pedro de Cisneros: "En catorse de enero [de 1493] Alonso de Porcuna. Diósele [...] un palacio alto e baxo que tenia tomado Pedro de Cisneros, que no le estava asentado, alinde del corral del dicho Alonso de Porcuna, e ha de dexar un cuerpo de casa que tenía tomado en la delantera, ecebto una salida que ha de sacar por donde quedó señalado por el rincón del palacio e por el otro rincón del portal por do está la viga de lo caído; e quedaron contentos las partes": LR, vol. II, 221.

13 Pedro de Cisneros, originario de Beas, se avecindó el 31 de agosto de 1487 declarando que traía diez mil maravedís de hacienda, y en febrero de 1489 recibía una casa conformada por dos corpezuelos, ordenándosele que cerrara "la puerta de la escalera del un alto" porque no entraba en la donación: LR, vol. I, 156. Efectivamente, ese era el palacio que ocupaba indebidamente en 1493, y los repartidores entregaron al albañil Alonso Ramos.

14 El 10 de octubre de 1489 se donó esta casa a Sebastián de Vega, escudero, que fallecía poco después "y se tuvo ynformaçión que no es casado", por lo que la casa quedó vacante. No obstante, pocos meses después su mujer Elvira Martínez -o Muñoz- reclamaba las casas y los derechos de vecindad que le hubiera correspondido a su marido, emprendiendo un pleito por verse envuelta en ciertas denuncias que se resolvería en 1497 cuando los monarcas ordenaron "que la vecindad de Sebastián de Vega quede a su mujer y a los hijos que dejó, en atención a lo que sirvió en vida" adjudicándosele otra casa en la misma calle Adalides, enfrente 
de que en 11 de enero de 1496 se donase definitivamente a Rodrigo de Sopuerta, originario del valle homónimo de Vizcaya, escudero de a pie de la reina y uno de los treinta criados de Garcí Fernández Manrique, primer corregidor de Málaga ${ }^{15}$. En octubre de 1489 se la describía como "una casa que es en una barrera de la cal de Adalides, con un pedaço de un corral que está a las espaldas, çerrando de pared por el postrimer çimiento de una paredeja derribada, paneando por su hilo derecho"16.

Es bastante probable que entre la casa de Alonso de Porcuna y la de Rodrigo de Sopuerta existiese una estrecha calleja, y así lo hemos dibujado en el plano, dado que ambos vecinos compartían linderos con Alonso Ramos y con Pedro de Cisneros ${ }^{17}$, pero hemos de señalar que la descripción de estas parcelas resulta muy confusa. De lo que no cabe duda es que existía una calleja al otro lado, entre las casas de Rodrigo de Sopuerta [Mártires, 6] y la de Arias Pinto [Mártires, 4], calleja que se adentraba en el interior de la manzana y salía a la calle Guardas, por lo que la denominamos barrera Adalides-Guardas, donde vivían maestre Pedro Alemán, lombardero y artillero avecindado el 31 de agosto de $1487^{18}$, y Alonso Fernández de Córdoba, peraile y odrero, originario de Córdoba, avecindado en febrero de $1489^{19}$. En el rincón de esta calleja tenía una de sus entradas las casas que pertenecían a Arias Pinto: "Junto con ésta [Alonso Fernández], en el dicho rincón, se vido la casa de Arias Pynto, que tiene su puerta principal a la calle de las Guardas"20.

\section{Mártires, 2-4 y Compañía, 10 (Palacio de Villalón).}

El beneficiario del solar donde se encuentra el Palacio de Villalón fue Arias Pinto, portugués vinculado a la casa ducal de Braganza, que junto a otros portugueses exiliados en Castilla tras la decapitación del duque Fernando II, se beneficiaron de mercedes

de la Iglesia de los Mártires: LR, vol. I, 134; vol. II, 150; vol. III, 432; vol. V, 166 y 272; AGS, RGS, marzo, 1490, fol. 248. De cualquier forma, tras el fallecimiento de Sebastián de Vega la casa que nos ocupa se donó a Andrés de Covarrubias, criado y pariente del obispo de Ávila, que también acabó perdiéndola por no residir en la ciudad "con muger e casa poblada el tiempo que sus altesas tyenen mandado e ordenado", entregándose entonces la casa a Rodrigo de Sopuerta: LR, vol. I, 134; vol. II, 242.

15 AGS, RGS, diciembre, 1491, fol. 250.

16 LR, vol. I, 134.

17 De cualquier forma, la pequeña barrera se cerraría tempranamente, si no lo hizo ya en estos momentos, dado que los repartidores ordenaron cerrar numerosas callejas que habían perdido su funcionalidad en la nueva concepción urbanística implantada por los castellanos, pasando el espacio de esas callejas a engrosar el parcelario de las casas adyacentes.

18 LR, vol. I, 414. Maestre Pedro recibió "un cuerpo de casa" en 20 de marzo de 1490, acrecentándosele poco después un corralejo: LR, vol. I, 162.

19 LR, vol. I, 436. La donación de su casa se efectuó el 19 de febrero de 1489: "Este dicho día se dio a Alonso Ferrández, perayle, en una barrera de cal de Adalides, una casa, la frontera del cabo de la barrera, en que ay un cuerpo, e que de otro corpezuelo de las espaldas se verá lo que se le deva dar para con ella": LR, vol. I, 162.

20 LR, vol. II, 147. Cabe señalar que en una de las intervenciones realizadas en las zonas de las casas 4,6 y 8 de la calle Mártires se descubrieron niveles de dos casas almohades y nazaríes (siglos XII-XV), apareciendo las estructuras características de los patios centrales árabes, con sus crujías y sus fuentes: GUILLÉN, A., "Treinta enterramientos de época bizantina", La Opinión de Málaga, 6 de noviembre de 2009, disponible en http://www. laopiniondemalaga.es/luces-malaga/2009/11/07/treinta-enterramientos-epoca-bizantina/300799.html. 
otorgadas por los Reyes Católicos ${ }^{21}$. Arias Pinto, que a la sazón era ayo de don Jaime y don Dionis, hijos del duque de Braganza, había llegado a Castilla en 1484 en compañía de su mujer e hijas ${ }^{22}$, residiendo primero en Sevilla en una casa otorgada por merced de los Reyes Católicos, desde donde continuaba prestando sus servicios a la casa ducal ${ }^{23}$. Unos años después, a principios de 1489, hizo acto de presencia en Málaga portando una cédula de la reina Isabel fechada en 13 de abril de 1488 por la que se le hacía merced de "unas buenas casas que para él convengan", ordenando también los monarcas que se le considerara como miembro del grupo de "las diez partes", incluyéndose así entre los principales de la ciudad. De acuerdo con su estatus, Arias Pinto recibía, en noviembre de 1489, una casa en la calle de los Adalides (Mártires, 2 y 4) y otra en la cal de las Guardas (Compañía, 10), que con anterioridad habían ocupado otros vecinos ${ }^{24}$.

"Este dicho día dieron por çédula de sus altezas a Arias Pinto la casa de Gamboa, que es a la calle de los Adalides, e otra en que bivía Bartolomé Sánchez de Alcabdete, que es en la cal de las Guardas al pozo dulçe, con todo lo que en ella se está eçebto otro cuerpo que estaba metido con las dichas casas de Ganboa. Testigos los dichos. Diéronle más el dicho cuerpo que es donde está un çidro en un patyn".

Es bastante probable que poco después se le añadiera la casa-algorfa o casillasoberado que en la actualidad atraviesa la calle de los Mártires, que en un principio se había donado a Juan de Cañete, labrador:

"En diez e nueve de março deste dicho año [1490] se dieron a Juan de Cañete, labrador, unas casas que son a la calle de los Adalides, en que ay un cuerpo pequeño con otras casillas soberados que llegan desde la esquina de la barreruela de las casas de Arias Pinto fasta la plaçuela derribada del pozo, con tanto que derribe el palaçio del primero corpezuelo quedando por frontero la pared de la puerta del dicho palaçio e ensanchando la calle por donde le está señalado"25.

Por motivos que ignoramos, Juan Ruiz de Cañete terminó viviendo en la calle Beatas $^{26}$, y pensamos que la casilla-soberado acabó uniéndose a las propiedades de Arias

21 Sobre la presencia de portugueses en el Reino de Granada a finales del siglo XV, LÓPEZ BELTRÁN, Ma T., y GONZÁLEZ ARÉVALO, R., "Los portugueses en el Reino de Granada en época de los Reyes Católicos (Málaga, 1487-1518)", Baetica, $n^{\circ} 24,2002$, pp. 309-338, donde se analiza de manera particular el grupo de portugueses vinculados a la nobleza portuguesa exiliada en Castilla que se asentaron en la ciudad de Málaga a finales del siglo XV.

22 En unas instrucciones emanadas de Juan II de Portugal fechadas en 15 de junio de 1484, respondiendo a demandas de Isabel la Católica, se dice: "quanto a o don Arias Pinto, lhe direes que ja o despachamos e a sua mulher et filhas", recogido en DE LA TORRE, A., "Los hijos del Duque de Braganza en Castilla (1483-1496)", Hidalquía, n 50, CSIC, Madrid, 1962, pp. 161-168.

23 AGS, RGS, febrero, 1484, fol. 11; octubre, 1484, fol. 38; marzo, 1487, fol. 50; junio, 1488, fol. 225.

24 LR, vol. I, 110, 263; vol. V, 145.

25 LR, vol. I, 214

26 LR, vol. II, 210. 
Pinto porque durante la visitación de 1493 su casa quedó pendiente de confirmación hasta revisar "el libro y su asiento" al hallarse "muchos cuerpos de casas", quedando finalmente confirmada la donación en abril de $1494^{27}$. Arias Pinto conseguía, por tanto, unas buenas casas valoradas en 100.000 maravedís ${ }^{28}$, situadas entre las calles Adalides y Guardas, con fachada principal a esta última calle, en el rincón que conformaba la "plazuela del pozo dulçe", frente a la actual calle Salvago, que precisamente se abrió a finales de $1488^{29}$, todo lo cual nos describe el emplazamiento de la Casa-Palacio de los Villalón.

\section{Compañía, 8 y 6.}

Siguiendo nuestro recorrido, junto a la casa de Arias Pinto se hallaba la casa que habían dado en 1489 a Garci Vázquez de Lora [Compañía, 8]:

"Este dicho dia [29-9-1489] se dieron a Garçi Vazques de Lora, escudero, unas casas que son en la calle de las Guardas en que ay un cuerpo bueno de casas e otro pequeño razonable, con una casillas soberados junto con ellas, fasta la esquina del pozo, de que son linderos de la una parte casas de maestre Hance el çiego, e de la otra parte a las espaldas casas de Arias Pinto, e la plaçuela del dicho pozo"30.

En la visitación de 1493 la casa de Garci Vázquez también quedó pendiente de confirmación porque, según aducían los repartidores, no se ajustaba a la categoría del beneficiario, pues siendo "recuero y trabajador" se había inscrito en el grupo de los escuderos. Y efectivamente, hemos constatado que en el avecindamiento del día 29 de agosto de 1487 consta el registro de "Garci Vázquez, vecino de Lora, escudero" 31 , pero lo cierto fue que conservó las casas porque poco después los repartidores confirmaron su donación añadiendo la consabida frase "quédale según su asiento y donación". Es posible que la donación se le confirmase por una carta que los monarcas habían remitido a los repartidores en julio de 1489, ordenándoles que concedieran alguna mejora en las casas y hacienda que señalasen a García Vázquez, el cual estuvo en la ciudad durante la peste "de la cual murieron su mujer y un hijo"32.

Junto a García Vázquez se hallaba la casa de maestre Hance, lombardero [Compañía, 6], que tampoco se libró de problemas durante la visitación, ya que "no se falló asiento porque es de merced", aunque todo se solucionó favorablemente en abril de 1494 siéndole confirmada su donación ${ }^{33}$. Por último, sabemos que maestre Hance

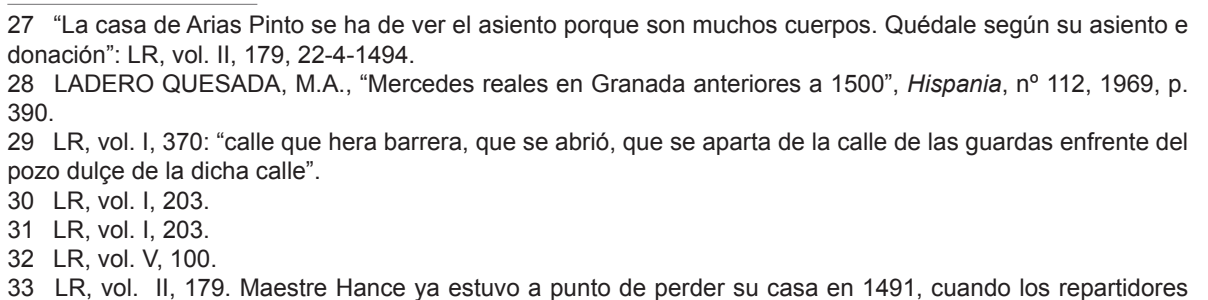


acabó vendiendo la casa a Antón López de Toledo, escribano público que actuó en la reformación del Repartimiento, y éste la incluyó entre los bienes dotales que otorgó a su hija Isabel López cuando en 1501 fue comprometida con don Luis Fajardo, hijo del regidor Diego Fajardo, linaje oriundo del reino de Murcia $^{34}$.

\section{Compañía, 12, y Plaza de San Ignacio, 4.}

El solar donde se asienta el edificio Administrativo y de Servicio del Museo eran las casas que se adjudicaron a finales de 1491 a Juan de Alcaraz, contino de la casa real y hermano del repartidor Francisco de Alcaraz, que constaban de "unos altos ençima de la entrada, e un cuerpo bueno de casa, e otro más pequeño" a las que se habían añadido otras casas a sus espaldas a las que se accedía por una barrera de la calle de los Adalides"35. La calleja en cuestión, que se adentraba por los actuales números 3 y 5 de la calle de los Mártires y salía a la actual plaza de San Ignacio, acabó también incorporándose a las casas de Juan de Alcaraz, y cabe añadir que en esa misma calleja, en el espacio que hoy se conforma delante de la Iglesia del Sagrado Corazón, en la plaza de San Ignacio, se hallaba una mezquita que se incluyó entre los bienes dotales de la Iglesia de Málaga: "junto con esta mesquyta está una calleja en medio, que se ha de cerrar e la ha de tomar Juan de Alcaraz"36. Resta añadir que a mediados de 1501 el contino Juan de Alcaraz abandonaba precipitadamente la ciudad huyendo de las actuaciones del Tribunal de la Inquisición que se había establecido en Granada a finales de 1498, constando ya en agosto de 1501 como vecino de Toledo tras haber vendido sus casas de Málaga por 52.000 maravedís al mercader judeoconverso Fernando de Córdoba ${ }^{37}$. Años

la entregaron a otro vecino porque el lombardero se ausentó de la ciudad: "Este dicho día [28-3-1491] se dio donaçión a Luis de Baeça, escudero, de las casas que tenía maestre Hançe en la calle de las Guardas, por quanto el dicho maestre Hançe se fue a otro reyno e no está aquí, en que ay tres cuerpezuelos, dos pequeños e el uno razonable; pero que si el dicho maestre Hançe viniere dentro de tres meses primeros syguientes, le volverá las dichas casas": LR, vol. I, 243.

34 Al respecto véase LÓPEZ BELTRÁN, $M^{a} \mathrm{~T}$. "Perfil de un judeoconverso del reino de Granada: el escribano Antón López de Toledo (149-1516), Revista del Centro de Estudios Históricos de Granada y su Reino, $n^{\circ} 18$, 2006, 53-76.

35 LR, Vol. I, 224. Como ocurrió en otras muchas ocasiones, las casas que acabaron siendo de Juan de Alcaraz se habían dado antes a otros repobladores que, por diversos motivos, perdieron la vecindad o bien se les adjudicaron otras viviendas: el primero en ocuparlas fue Pedro de Aguilar, labrador aragonés avecindado en Málaga el 30 de agosto de 1487, a quien los repartidores dieron otra casa en la calle "Monteros", actual calle Alcazabilla: LR, vol. I, 458; vol. II, 104. Luego ocupó la casa el bachiller Alonso Escudero, caballero de las "siete partes", que fue el primer alcalde mayor nombrado por el corregidor Garcí Fernández Manrique actuando en el cargo entre los años 1489-90: RUIZ POVEDANO, J. Ma.., El Primer Gobierno Municipal de Málaga (1489-1495), Granada, 1991, 157-158. Sin embargo, a finales de 1491 Alonso Escudero perdía la casa y todos los bienes que había recibido por repartimiento por no cumplir los plazos que se le dieron para venir a residir a la ciudad con su mujer, incluso habiéndosele prorrogado dos meses más "porque dixo que su muger está para paryr e no podrá venir de aquí a dos meses": LR, vol. I, 486, 503. También la casa que añadieron a las espaldas de la casa principal había pertenecido antes a otro vecino, Juan de Gámez, sastre, que acabó residiendo en la calle "Mercaderes", actual Santa María: LR, vol. I, 225.

36 LR, vol. II, 161. Sobre la situación de las mezquitas de Málaga en época musulmana, GARCíA RUIZ, Mª V., Málaga..., 95-111.

37 LÓPEZ BELTRÁN, Ma T., "Los inicios de la Inquisición en Málaga y su obispado", Chronica Nova, n 30, 
después, en agosto de 1511, el mercader Fernando de Córdoba revendía estas casas al regidor Agustín Ytalian, por valor de 85.000 maravedís $^{38}$.

\section{MÁrtires, 5.}

En el solar donde se ubica la Sede de la Fundación del Museo se hallaban las casas que dieron a Juan Fernández de Ávila, organista -conocido también como Juan Dávila-y Francisco de Sevilla, tejedor. La casa del organista, donada en febrero de 1489, constaba de "dos cuerpos solos"39, y similar debía ser la vivienda que dieron a Francisco de Sevilla cuando se avecindó en marzo de 1490, aunque quizá se hallaba en peores condiciones porque tenía "un cuerpo grande derribado a las espaldas", colindando con la mezquita ya mencionada que también lindaba con las casas de Juan de Alcaraz ${ }^{40}$.

Antes de su integración en el conjunto museístico, el solar lo ocupaba una antigua fábrica de cera que ha conservado su maquinaria, rehabilitándose la vivienda, que aún mantiene muros del siglo XV y la estructura general mudéjar con su pequeño patio central, recuperándose también armaduras interesantes, ya más tardías, y las pinturas de la fachada que envuelven sobre todo la bella galería del piso superior ${ }^{41}$.

Actualmente, el sistema viario que conforma el entorno de las calles Compañía, Mártires y vías aledañas sigue guardando los rasgos heredados de la época musulmana y las aportaciones urbanísticas que se llevaron a cabo durante la primera etapa de experiencia repobladora. En aquellos momentos, el ámbito de la calle de las Guardas ya empezaba a distinguirse acogiendo a personajes selectos de la sociedad repobladora. Por citar algunos ejemplos, en aquel entorno recibieron casas por merced tres miembros del Consejo Real: don Enrique Enríquez, mayordomo mayor del rey Católico y miembro del Consejo Real ${ }^{42}$, cuya casa ocupaba el solar de lo que hoy se conoce como antiguo Parador de San Rafael, en la Plaza de Puerta Nueva, que hace poco fue declarado Bien de Interés Cultural. También don Álvaro de Portugal, noble de la casa ducal de Braganza y Presidente del Consejo Real de

2003-2004, 230. Así, mismo, sobre el mercader Fernando de Córdoba, LÓPEZ BELTRÁN, Ma T., “El poder económico en Málaga: la familia Córdoba-Torres (1493-1538)", en LÓPEZ DE COCA CASTAÑER, J.E. y GALÁN SÁNCHEZ, A. (coords.), Las ciudades andaluzas (siglos XIII-XVI). Actas del VI Coloquio Internacional de Historia Medieval de Andalucía, Málaga, 1991, pp. 463-482.

38 LÓPEZ BELTRÁN, Ma T., "El poder económico...", 481; LÓPEZ DE COCA CASTAÑER, J. E., y LÓPEZ BELTRÁN, M ${ }^{a}$ T., "Mercaderes genoveses en Málaga (1487-1516): los hermanos Centurión e Ytalian", Historia, Instituciones, Documentos, $\mathrm{n}^{\circ} 7,1980$, pp. 95-124.

39 LR, vol. I, 156.

40 Francisco de Sevilla, que figura inscrito en el avecindamiento el mismo día que recibió la donación de su vivienda, era originario de Fuente Salida y declaró que traía 30.000 maravedís de hacienda: LR, vol. I, 214 y 423.

41 CAMACHO, R. (Dir.), Guía..., 345.

42 LR, vol. I, 107 
los Reyes Católicos, recibió casas por merced ubicadas al principio de la calle Pozos Dulces $^{43}$. Y don Rodrigo Maldonado, más conocido como doctor de Talavera, recibió casas por merced en una barrera de la calle de las Guardas ${ }^{44}$, en el suelo donde en la actualidad se levanta la Iglesia del Sagrado Corazón, en la Plaza de San Ignacio.

La apertura de la Puerta Nueva en 1494 supuso, sobre todo, un cambio en la función económica del aquel sector urbano de la ciudad, que "se ennoblesçería muncho por el trato de la Plaça e Puerta"45, lo que justifica que en la calle de las Guardas o de San Sebastián, como sería conocida poco después, se fueran estableciendo hombres de negocios y de reconocida capacidad económica, como el mercader Fernando de Córdoba, del que ya nos hemos ocupado con anterioridad. También, andando el tiempo, se instalaría en esta calle la Compañía de Jesús, dándole el nombre que conserva en la actualidad.

No cabe duda que el Museo Carmen Thyssen Málaga ha supuesto un hito importante en la rehabilitación del espacio que ocupa y, por tanto, del centro histórico de la ciudad. Pero también ha servido, como en este caso, para retrotraernos a nuestro pasado histórico a través de la herencia urbanística musulmana y cristiana y de las familias que conformaban el vecindario de Málaga a finales del siglo XV.

43 LR, vol. I, 135.

44 LR, vol. II, 161.

45 POVEDANO RUIZ, J. Mª., Málaga, de musulmana a cristiana, Málaga, 2000, 267. 
